\title{
Statistical performance evaluation and comparison of a Compton medical imaging system and a collimated Anger camera for higher energy photon imaging
}

\author{
Li Han ${ }^{1}$, W Leslie Rogers ${ }^{1,2}$, Sam S Huh ${ }^{1}$ and Neal Clinthorne ${ }^{2}$ \\ ${ }^{1}$ Department of Biomedical Engineering, University of Michigan, Ann Arbor, MI, USA \\ ${ }^{2}$ Division of Nuclear Medicine, University of Michigan, Ann Arbor, MI, USA \\ E-mail: 1han@umich.edu
}

Received 24 June 2008, in final form 24 September 2008

Published 18 November 2008

Online at stacks.iop.org/PMB/53/7029

\begin{abstract}
In radionuclide treatment, tumor cells are primarily destroyed by charged particles emitted by the compound while associated higher energy photons are used to image the tumor in order to determine radiation dose and monitor shrinkage. However, the higher energy photons are difficult to image with conventional collimated Anger cameras, since a tradeoff exists between resolution and sensitivity, and the collimator septal penetration and scattering is increased due to the high energy photons. This research compares imaging performance of the conventional Anger camera to a Compton imaging system that can have improved spatial resolution and sensitivity for high energy photons because this tradeoff is decoupled, and the effect of Doppler broadening at higher gamma energies is decreased. System performance is analyzed by the modified uniform Cramer-Rao bound (M-UCRB) algorithms based on the developed system modeling. The bound shows that the effect of Doppler broadening is the limiting factor for Compton camera performance for imaging $364.4 \mathrm{keV}$ photons emitted from ${ }^{131} \mathrm{I}$. According to the bound, the Compton camera outperforms the collimated system for an equal number of detected events when the desired spatial resolution for a $26 \mathrm{~cm}$ diameter uniform disk object is better than $12 \mathrm{~mm}$ FWHM. For a 3D cylindrical phantom, the lower bound on variance for the collimated camera is greater than for the Compton imaginer over the resolution range from 0.5 to $2 \mathrm{~cm}$ FWHM. Furthermore, the detection sensitivity of the proposed Compton imaging system is about 15-20 times higher than that of the collimated Anger camera.
\end{abstract}

(Some figures in this article are in colour only in the electronic version) 


\section{Introduction}

High-resolution imaging for $\gamma$ rays with energies above $250 \mathrm{keV}$ such as those emitted by ${ }^{131} \mathrm{I},{ }^{113 \mathrm{~m}} \mathrm{In},{ }^{137} \mathrm{Cs}$ or ${ }^{22} \mathrm{Na}$ could well become clinically useful for diagnosing cancer, studying physiological and chemical processes of various tumors, monitoring tumor therapy, and tracking the metabolic activity of essential trace elements. These radionuclides and corresponding radiopharmaceuticals cannot be replaced by low-energy radiotracers because they have unique chemical properties that make them organ and/or tumor specific. For example, ${ }^{131}$ I or radiotracers labeled with it can be used for both diagnostic imaging and internal radiotherapy to the thyroid gland. ${ }^{131} \mathrm{I}$ primarily emits detectable gamma-rays at $284 \mathrm{keV}(6.05 \%), 364.4 \mathrm{keV}(81.2 \%), 636 \mathrm{keV}(7.26 \%)$ and $723 \mathrm{keV}(1.8 \%)$; it also emits $\beta^{-}$particles for cancer radiotherapy. During ${ }^{131} \mathrm{I}$ radiotherapy, it is necessary to employ an effective imaging system to determine radiation dose and monitor tumor shrinkage.

Imaging performance of conventional collimated Anger camera systems (Anger 1958, 1964 ) for detecting $\gamma$-ray photons is primarily determined by properties of the collimator. An Anger camera system with a parallel-hole high energy general purpose lead collimator (HEGP) imposes an efficiency-resolution tradeoff because of the physical constraints resulting from the mechanical collimation. As the imaged $\gamma$-ray photons exceed $\sim 250 \mathrm{keV}$, the collimator septal thickness must be increased to reduce the penetration and scattering of higher energy photons in the collimator material. Since collimator sensitivity is reduced as the square of hole diameter, resolution must be sacrificed by increasing hole size if sensitivity is to be maintained. Moreover, since the photoelectric cross section of material decreases as the cube of incoming photon energy, the sensitivity of the collimated Anger camera is also reduced when imaging higher energy photons.

Compared to the collimated Anger camera, the Compton imaging system (Todd 1974, Singh 1983, Solomon and Ott 1988, Philips 1995, Leblanc 1998, 1999) is an image formation technique that has the potential for significantly improved performance in both detection efficiency and spatial resolution for imaging higher energy photons even though at $140 \mathrm{keV}$ (Hua 1999), a ring Compton imaging system requires at least 40 times the sensitivity of an Anger camera with low energy general purpose lead collimator to achieve similar imaging performance. According to the basic principle of Compton scattering (Compton 1923), an incident $\gamma$-ray photon with energy $E_{0}$ scatters from and transfers part of its energy $E_{1}$ to an electron in the first detector. If the transferred energy exceeds the electron's binding energy, the electron will be released from the atom. The photon with reduced energy $E_{2}$ is scattered at a scattering angle $\theta$ with respect to its initial direction, and finally absorbed in the second detector. If the initial electron is assumed to be free and at rest, following the conservation laws of energy of momentum (Evans, 1982, Knoll, 2000), the relationship between the scattered photon energy $E_{2}$ and the scattering angle $\theta$ is expressed as

$$
\cos \theta=1+m_{0} c^{2}\left(\frac{1}{E_{0}}-\frac{1}{E_{2}}\right),
$$

where $m_{0} c^{2}=511 \mathrm{keV}$ is the rest energy of the electron.

Using the positions and energies of the interaction in time-coincidence in the first and second detectors in conjunction with the above Compton kinematics expression, each incident photon can be localized within a conical ambiguity in image space. Measurements recorded from an ensemble of incident photons can be used to reconstruct and estimate the source distribution. In essence, the Compton system uses 'electronic collimation' instead of absorbing collimation to form an image and has the potential for improving detection efficiency and 
spatial resolution simultaneously by eliminating the efficiency-resolution tradeoff imposed by the conventional collimator.

In the real case, equation (1) is an oversimplification: electrons in the scattering detector are neither free nor at rest. The random momentum distribution of electrons introduces a corresponding uncertainty or blur into the scattering angle associated with a given deposited energy. This blurring is referred to as Doppler broadening (Ordonez et al 1997). Due to the uncertainty in the recoil electron energy measurement and the effect of Doppler broadening in the first detector combined with the intrinsic spatial resolution of the first and second detectors, the scattering angle is not precisely determined and the source location is known only within a conical surface with finite angular thickness. In a well-designed Compton camera, spatial resolution is principally influenced by Doppler broadening and finite energy resolution of the scattering detector. Nevertheless, a Compton camera still offers the potential for a joint improvement in image noise and spatial resolution compared to mechanically collimated cameras because the greatly increased sensitivity can overcome the reduced information carried per detected photon. Imaging high energy $\gamma$-ray photons, as we propose, substantially reduces the influence of Doppler broadening and detector noise resulting in the potential for further improving the spatial resolution.

This research compares the theoretical imaging performance of a dual-planar siliconbased Compton imaging system with that of an Anger camera with HEGP collimator using statistical analysis tools, and the modified uniform Cramer-Rao bound (M-UCRB). The bound was proposed to compare the minimum achievable variance in reconstructed images at a given target spatial resolution defined as a symmetric point-spread function (PSF) specified by the desired full width at half-maximum (FWHM). For this particular medical application, we are interested in diagnostic imaging tracers and therapeutic agents labeled with ${ }^{131}$ I that emits predominately $364.4 \mathrm{keV}$ energy photons. The choice of energy not only corresponds to a medically important radionuclide but is also in a regime where the performance of conventional collimation is rapidly decreasing while that of Compton collimation is rapidly increasing.

\section{Methods and algorithms}

In order to compare performance between imaging systems, both a well-defined task and a figure-of-merit for accomplishing that task must be specified. For this study we focus on the ability to quantify activity at given point in the object. For such a task in the non-Bayesian estimation setting, the two most important performance parameters are the variance of the estimate (i.e., noise in the reconstructed images), and its bias or systematic error. For a given radioactivity distribution, variance is introduced by the conditional Poisson measurement noise in combination with the resolution recovery or reconstruction procedure. Bias, on the other hand, can be the result of incompletely removing the effects of finite spatial resolution or it can be introduced intentionally in order to stabilize variance. For example, smoothing a reconstructed image can decrease noise at the expense of increasing bias and degrading the image resolution. The conditional mean-squared error (MSE) of an estimate is the sum of the variance and the square of the bias. While zero bias or perfect resolution may be desirable, reducing bias typically increases variance leading to a tradeoff. Moreover, minimum MSE is typically not obtained at zero bias and it is therefore important to characterize performance over a range of bias or, more correctly, a range of bias gradients.

Since absolute bias is highly object dependent, we compare systems on the basis of their 'potential' for bias as quantified through a desired point-spread function (PSF) or local impulse response in reconstructed images and their variance at each width of PSF. Accordingly, each imaging system will exhibit a corresponding noise-resolution tradeoff curve. Although not 
often used outside of tomography, resolution can also be recovered in planar scintigraphy and in performing system comparisons, it is important that this be taken into account. A system that achieves lower noise than another irrespective of desired resolution (as referenced through the PSF), is uniformly better. Nevertheless, it may well be that one system is better than another only over a specific range of resolution in reconstructed images.

It is desirable to compare system performance based only on the intrinsic quality of their measurements and not with the confounding influence of image reconstruction or postprocessing procedures, which are easily changed. To this end, we use the fact that the limiting variance of any unbiased estimator or reconstruction method is lower bounded by the inverse of the Fisher Information Matrix (FIM) also known as the Cramér-Rao bound (CRB) (Kay 1993, Van Trees 2001). As noted above, unbiased estimation is not necessarily a desirable attribute and we employ a modification to the uniform CRB introduced by Usman and Hero (Usman 1994, Hero 1996) to quantify the performance of biased estimators. In the modified uniform CRB (M-UCRB) introduced by Meng and Clinthorne (Meng 2004) and detailed in section 2.1.1, the variance for each imaging system is compared at essentially the same target PSF (or more accurately, mean estimator gradient) in reconstructed images and traced as a function of the width of the target PSF (e. g., FWHM of a Gaussian response). Although, the M-UCRB provides a lower bound on the variance independent of any reconstruction or resolution recovery procedure, it is important to note that use of the penalized maximumlikelihood estimate that has been post-smoothed by the desired PSF asymptotically achieves the limiting variance.

The M-UCRB requires calculation of the FIM and inversion of large matrices. For imaging systems that have a high dimensional measurement space-in particular, Compton camera measurements are indexed by energy and by first and second detector locationsdirect calculation can be computationally prohibitive. Here, Monte Carlo integration (MCI) methods (Press et al 2002, Meng and Wehe 2003, Parra and Barrett 1998) are used to calculate the FIM and are described in section 2.1.2. Inversion of the large matrices necessary to compute the M-UCRB is accomplished by assuming that the response of each imaging system is approximately shift-invariant. Accordingly, the fast Fourier transform (FFT) can be used to reduce computational complexity from $\mathrm{O}\left(N^{3}\right)$ to $\mathrm{O}(N \log N)$ where $N$ is the dimension of FIM as detailed in section 2.1.3. Finally, sections 2.2 and 2.3 describe how the two imaging systems are modeled for the performance calculations.

\subsection{Modified Uniform Cramer-Rao Bound}

2.1.1. Background. The M-UCRB imposes a constraint on the mean gradient of the estimator $\nabla_{\underline{\theta}} m_{\underline{\theta}}\left(\hat{\theta}_{j}\right)$, and is

$$
\begin{aligned}
\nabla_{\underline{\theta}} m_{\underline{\theta}}\left(\hat{\theta}_{j}\right) & =\nabla_{\underline{\theta}} b_{\underline{\theta}}\left(\hat{\theta}_{j}\right)+\underline{e}_{j} \\
& =\left[\frac{\partial E_{\underline{\theta}}\left(\hat{\theta}_{j}\right)}{\partial \theta_{1}}, \frac{\partial E_{\underline{\theta}}\left(\hat{\theta}_{j}\right)}{\partial \theta_{2}}, \ldots, \frac{\partial E_{\underline{\theta}}\left(\hat{\theta}_{j}\right)}{\partial \theta_{p}}\right] .
\end{aligned}
$$

The mean gradient describes the sensitivity of a single reconstructed pixel to the perturbations in true parameter values. Under the conditions (Fessler and Rogers, 1996, Kragh, 2002), that the mean of the estimator is $E_{\underline{\theta}}[\underline{\hat{\theta}}]=L \underline{\hat{\theta}}$ and the matrix $L$ is symmetric, the mean estimator gradient at the $j$ th pixel is close or equal to the PSF of the $j$ th pixel. The PSF specifies the influence of a perturbation of a single source parameter on all estimated pixel values in the reconstruction. Stated another way, the mean estimator gradient for the $j$ th pixel is give by a row of $L$ while the PSF at the $j$ th pixel is a column of $L$. 
By using the M-UCRB, the Euclidean norm of error vector between a desired mean gradient vector $f$, i.e. the target PSF and the actual PSF $g$ are less than a small pre-specified tolerance $\delta$, is given as

$$
\|g-f\| \leqslant \delta \quad \text { and } \quad \delta=\left\|\lambda\left[F_{Y}+\lambda I\right]^{-1} \cdot f\right\| .
$$

From the biased Cramer-Rao bound, that is

$$
\begin{aligned}
\operatorname{Var}\left(\hat{\theta}_{j}\right) & \geqslant\left[\nabla_{\underline{\theta}} E_{\underline{\theta}}\left(\hat{\theta}_{j}\right)\right]^{T} F_{\underline{\underline{Y}}}^{-1}\left[\nabla_{\underline{\theta}} E_{\underline{\theta}}\left(\hat{\theta}_{j}\right)\right], \\
& =g^{T} \cdot F_{\underline{\underline{Y}}}^{-1} \cdot g
\end{aligned}
$$

the optimal actual mean gradient is equal to

$$
\begin{aligned}
g_{\text {optimal }} & =\underset{\|g-f\| \leqslant \delta}{\arg \min }\left\{g^{T} \cdot F_{Y}^{-1} \cdot g\right\} . \\
& =[F+\lambda I]^{-1} \cdot F \cdot f
\end{aligned}
$$

Thus, the M-UCRB can be derived as

$$
\operatorname{Var}\left(\hat{\theta}_{j}\right)=\sigma^{2} \geqslant f^{T} \cdot\left[F_{Y}+\lambda I\right]^{-1} \cdot F_{Y} \cdot\left[F_{Y}+\lambda I\right]^{-1} f,
$$

where $F_{Y}$ is the Fisher information Matrix, $\lambda$ is a small positive scalar and $I$ is the identity matrix with same size as $F_{Y}$. Therefore, the lower bound of variance imposed by the target PSF can be calculated. It is meaningful to use the M-UCRB to evaluate imaging performance of different imaging systems by comparing the difference in variance in the image at the same PSF.

2.1.2. Monte Carlo calculation of FIM. The FIM-essentially the generalized signal-tonoise ratio of the measurements-is a key element for calculating M-UCRB, but calculation suffers from the computation and memory requirements for high dimensional images and measurements, such as the Compton imaging system. The conventional calculation of FIM for medical imaging systems is based on a statistical model of the detection process governed by Poisson statistics:

$$
\underline{Y} \sim \operatorname{Possion}(\Lambda A \underline{\theta}),
$$

where $A$ is the system response matrix, $\Lambda$ is the mean total number of events in the measurement interval, $\underline{Y}=\left[Y_{1}, \ldots, Y_{\mathrm{D}}\right]$, is a vector of the projection measurements, $\underline{\theta}=\left[\theta_{1}, \ldots, \theta_{\mathrm{P}}\right]^{\mathrm{T}}$ is the parameterized image space vector normalized as

$$
\sum_{i=1}^{D} \sum_{j=1}^{P} a_{i j} \theta_{j}=1
$$

Therefore, the FIM has the following form:

$$
F_{\underline{Y}}(\underline{\theta})=\Lambda \mathrm{A}^{\mathrm{T}}[\operatorname{diag}(A \underline{\theta})]^{-1} A .
$$

The inverse of the FIM provides a lower bound on the covariance matrix of any unbiased estimate of $\underline{\theta}$. To solve the calculation problem involved in directly calculating and inverting a non-sparse FIM, an alternative method is required to reduce the computational complexity and size of memory required to calculate the FIM and M-UCRB.

According to the definition of expected Fisher information, given the observable random variable $\underline{Y}$, unknown parameters $\underline{\theta}$ with $P$ elements, and conditional probability, $p_{\underline{Y}}(\underline{Y} \mid \underline{\theta})$, the $P \times P$ FIM is defined as

$$
F_{\underline{Y}}(\underline{\theta})=-E\left\{\frac{\partial^{2}}{\partial \theta^{2}} \log p_{\underline{Y}}(\underline{Y} \mid \underline{\theta})\right\}
$$


For the numerical calculation, the alternative method is referred to as the observed Fisher information (Berger, 1994), which is equivalent to using a Monte Carlo calculation. The one element with index of $i j$ in the FIM for $N$ observed random variables is given as

$$
\begin{aligned}
F_{i j} & =-\left\langle\frac{\partial^{2}}{\partial \theta_{i} \partial \theta_{j}} \log p_{\underline{Y}}(\underline{Y} \mid \underline{\theta})\right\rangle \\
& \approx-\frac{1}{N} \sum_{l=1}^{N} \frac{\partial^{2}}{\partial \theta_{i} \partial \theta_{j}} \log p_{\underline{Y}}\left(Y_{l} \mid \underline{\theta}\right)
\end{aligned}
$$

Where \langle\rangle denotes mean. According to the properties of the observed Fisher information, the $Y_{i}$ are independent and identically distributed, and the value of the element in the FIM for $\Lambda$ number of desired events is equivalent to $\Lambda$ times the value of the element in the observed FIM for a single desired event. This is given as

$$
F_{(\Lambda)_{i j}}=\Lambda \cdot F_{(1)_{i j}}
$$

Thus, the average value of one element of the observed Fisher information matrix may be calculated by a Monte Carlo Integration instead of using the implied multidimensional integration.

If the FIM corresponds to a desired mean number of detected events $\Lambda$, and $N$ is the fixed number of measured or simulated events for the Monte Carlo integration, the estimated value for one element in the observed Fisher information matrix is defined as

$$
\begin{aligned}
F_{\Lambda}(\underline{\theta})_{i j} & =\Lambda \cdot F_{1}(\underline{\theta})_{i j} \\
& \approx \frac{\Lambda}{N} \cdot \sum_{l=1}^{N} \frac{p\left(Y_{l} / i\right) p\left(Y_{l} / j\right)}{\left(\sum_{n=1}^{M} p\left(Y_{l} / n\right) \cdot f_{n}\right)^{2}}
\end{aligned}
$$

where $p\left(Y_{l} / i\right)$ is the probability that an event generated in source bin $i$ leads to a measurement $Y_{l}$ by the imaging system; $f_{n}$ is the expected number of photons emitted from source bin $n$, i.e. the object intensity in the $n$th source bin; and $M$ is the total number of source pixels.

Clearly, the estimated value of each entry in the FIM approaches the real value asymptotically and the variance of individual elements in FIM decreases with increasing $N$. Moreover, the calculation of observed Fisher information matrix by the Monte Carlo integration not only decreases the computational complexity to $M \times N$ floating point calculations for one element of the FIM but also requires only moderate system memory space.

2.1.3. Fast Fourier transform for M-UCRB estimation. Another primary computational issue of the M-UCRB is the inversion of the matrix $\left[F_{Y}+\lambda I\right]$, and multiplication of several matrices which have the same size as the FIM $F_{Y}$. Fortunately, if employing a source with uniform activity and assuming local spatial invariance, the matrices of both $F_{Y}$ and $\left[F_{Y}+\lambda I\right]$ are approximately circulant-block-circulant (CBC) matrices (Davis, 1994), in which the block rows are circular right shifts of the block elements of the preceding block row, and each block is still a circulant matrix.

According to the properties of block circulant matrices, the $\mathrm{CBC}$ square matrix $F$ or $F^{-1}$ multiplying a vector equals the first column of a CBC matrix cyclically convolved with the vector. This can be realized using a Fourier Transform. The DFT of an $N$-dimensional complex vector can be defined as matrix $W$, in which $W_{k n}=\left(\mathrm{e}^{-\left(\mathrm{i} \frac{2 \pi}{N}\right)}\right)^{k n}$. Thus, with unitary normalization constants $1 / \sqrt{N}$, the above DFT matrix is further defined by a unitary matrix: $Q=W / \sqrt{N}$. A corresponding IDFT unitary matrix is its Hermitian transpose matrix $Q^{*}$ and $Q \cdot Q^{*}=1$ and the circulant matrix $C$ can be separated as $C=Q^{*} \operatorname{diag}\left(Q C_{1 c}\right) Q$, where $C_{c}$ is the first column of $C$. 


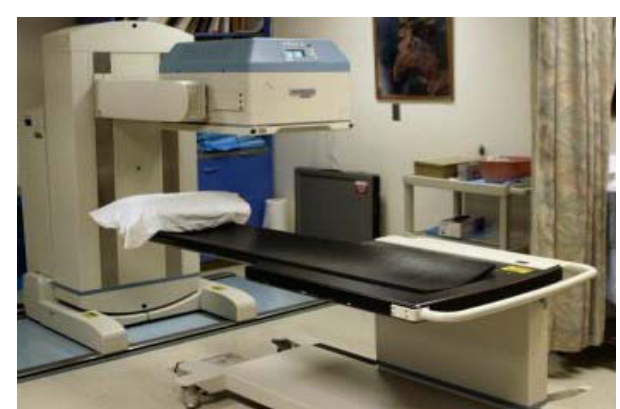

(a)

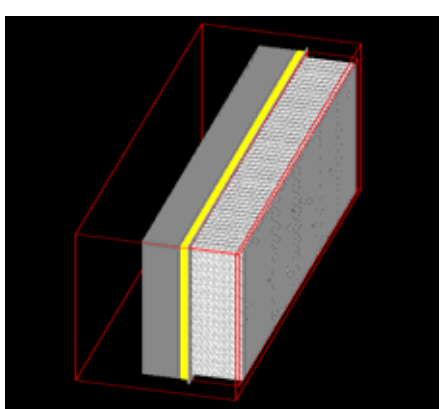

(b)

Figure 1. (a) The ADAC Lab ARGUS imaging system. (b) The simulated ARGUS camera head with parallel hole HEGP lead collimator (white represents lead collimator, yellow represents NaI crystal, and gray represents phototube array).

Therefore, the calculation of modified uniform Cramer-Rao bound is given as

$$
\begin{aligned}
\sigma^{2} & \geqslant f^{T}[F+\lambda I]^{-1} \cdot F \cdot[F+\lambda I]^{-1} f \\
& =f^{T} Q^{*}\left((Q f) \cdot /\left(Q[F+\lambda I]_{1 c}\right) \cdot{ }^{*}\left(Q F_{1 c}\right) \cdot /\left(Q[F+\lambda I]_{1 c}\right)\right),
\end{aligned}
$$

where '.*' and './' are element-wise multiplication and division, respectively.

\subsection{System modeling for anger camera with HEGP collimator}

2.2.1. Description of an Anger camera with HEGP collimator. The conventional Anger Camera used in this study is an existing commercial Anger camera head with high energy general purpose lead collimator, as shown in figure 1. The camera is part of the ARGUS imaging system manufactured by ADAC laboratories (ADAC).

According to the ARGUS system specifications, the NaI crystal thickness is $9.5 \mathrm{~mm}$ designed for a maximum energy of $400 \mathrm{keV}$, the intrinsic spatial resolution is $4 \mathrm{~mm}$ (FWHM), and intrinsic energy resolution is $10.6 \%$ (FWHM) for $364.4 \mathrm{keV}$. For detecting the $364.4 \mathrm{keV}$ photons from ${ }^{131} \mathrm{I}$, the hole size, septa and length of HEGP collimator are $3.81 \mathrm{~mm}, 1.727 \mathrm{~mm}$ and $60.0 \mathrm{~mm}$, respectively. The collimator spatial resolution is around $12.6 \mathrm{~mm}$ for a $364.4 \mathrm{keV}$ point source at $10 \mathrm{~cm}$ from the front face of the collimator. Figure 1(b), illustrates the Anger Camera with collimator model simulated by GATE (Geant4 Application for Tomographic Emission) and GEANT4 Monte Carlo simulation system (Jan et al 2004).

2.2.2. System Model of the Anger Camera with HEGP Collimator. In order to analyze the performance of a conventional Anger camera equipped with an HEGP lead collimator for imaging the high energy gamma rays from ${ }^{131} \mathrm{I}$, a system model of an Anger Camera with HEGP collimator for ${ }^{131} \mathrm{I}$ gamma rays which includes photon penetration and scattering in the collimator is required. The goal of this model is to find a relatively simple function to correctly describe the point-spread function at different distances from the image plane to the surface of the lead collimator. The model primarily considers the resolution properties of a parallel hole HEGP lead collimator and sodium iodide Anger camera.

Using the GATE simulation system, the point-spread function images at source distances from $0 \mathrm{~cm}$ to $50 \mathrm{~cm}$ from the front face of the collimator are generated for a point ${ }^{131} \mathrm{I}$ source in air; the energy window is $20 \%$ around $364.4 \mathrm{keV}$. The simulated ${ }^{131} \mathrm{I}$ point-spread function images and their profiles at distances of $10 \mathrm{~cm}$ are shown in figure 2(a). The circular average 


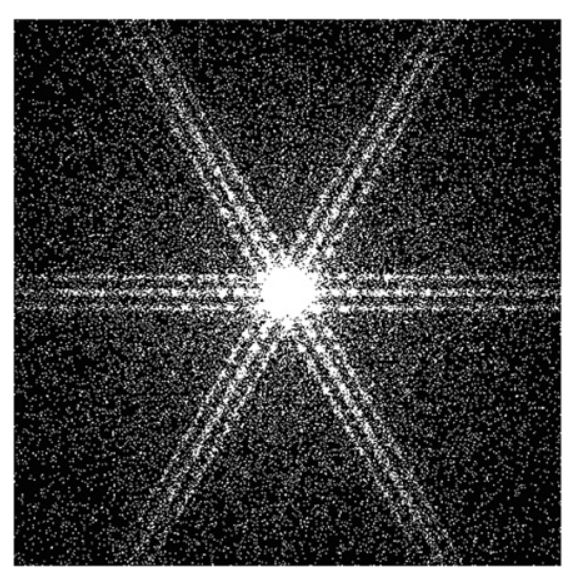

(a)

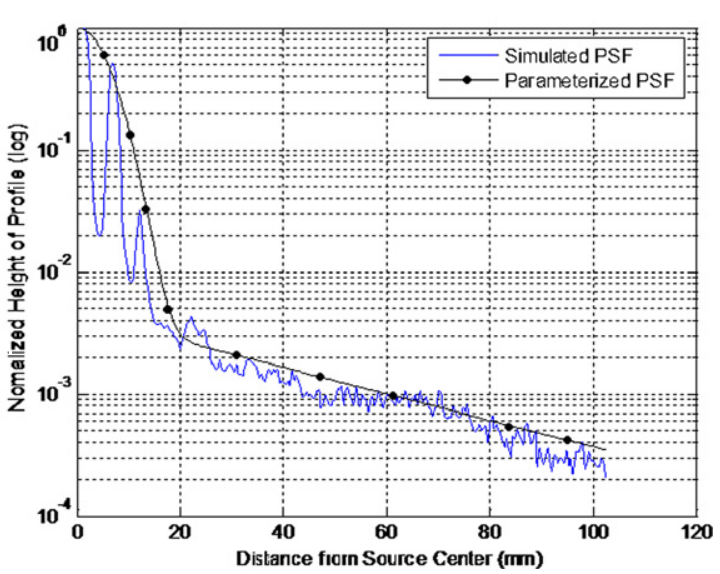

(b)

Figure 2. (a) The two-dimensional point-spread images for an $\mathrm{I}^{131}$ point source at a distance of $10 \mathrm{~cm}$. (b) Right half of simulated and fitted point-spread function for an $\mathrm{I}^{131}$ point source at a distance of $10 \mathrm{~cm}$.

of simulated PSF is then fit with a Gaussian combined with an exponential function that arises from collimator penetration and scattering by a nonlinear least-squares method. At a given distance $x$, the function is given as

$$
\operatorname{PSF}(x)=A_{\text {Gauss }} \mathrm{e}^{\left(\frac{-x^{2}}{2 \sigma^{2}}\right)}+A_{\text {exp }} \mathrm{e}^{(-\lambda x)} .
$$

As shown in figure 2(b), the right half profiles of the simulated normalized point-spread function and the fitted point-spread function from equation (15) are displayed for the ${ }^{131}$ I point source at $10 \mathrm{~cm}$. There is a good match between the original simulated PSF and the fitted PSF without consideration of the collimator hole pattern.

To derive the relationship of coefficients in the fitted point-spread function with point source to collimator distance, the simulated point-spread images were obtained at $0 \mathrm{~cm}, 1 \mathrm{~cm}$, $2 \mathrm{~cm}, 3 \mathrm{~cm}, 5 \mathrm{~cm}, 7 \mathrm{~cm}, 10 \mathrm{~cm}, 15 \mathrm{~cm}, 20 \mathrm{~cm}, 25 \mathrm{~cm}, 30 \mathrm{~cm}, 35 \mathrm{~cm}$ and $40 \mathrm{~cm}$, respectively. The value of coefficients $A_{\mathrm{Gauss}}, A_{\mathrm{exp}}, \sigma$ and $\lambda$ at different source to collimator distances and the curve obtained by nonlinear curve fitting. The final fitted functions for the four coefficients as a function of source to collimator distance $d$ are

$$
\begin{aligned}
& A_{\text {Gauss }}=0.03645 \times 10^{(-0.05967 \times d)}+0.007193, \\
& \sigma_{\text {Gauss }}=0.2768 \times d+2.454, \\
& A_{\exp }=0.002876 \times \mathrm{e}^{(-0.07266 \times d)}+0.0007339, \\
& \lambda_{\exp }=0.03877 \times 10^{(-0.04374 \times d)}+0.009312,
\end{aligned}
$$

\subsection{Statistical modeling for Compton imaging system}

2.3.1. Description of a dual-planar silicon-based Compton imaging system. As shown in figure 3(a), the scatter detector studied consists of $32 \times 16 \times 10$ silicon pad detector elements and each silicon pad is $1.4 \mathrm{~mm}$ by $1.4 \mathrm{~mm}$ by $1 \mathrm{~mm}$. Silicon pad sensors can be stacked 


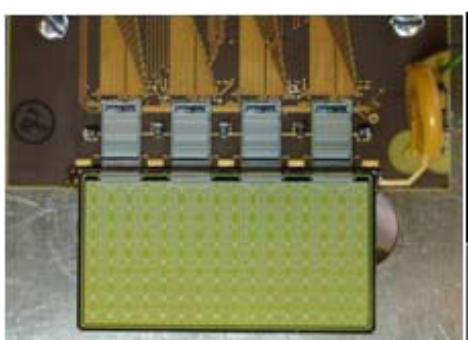

(a)

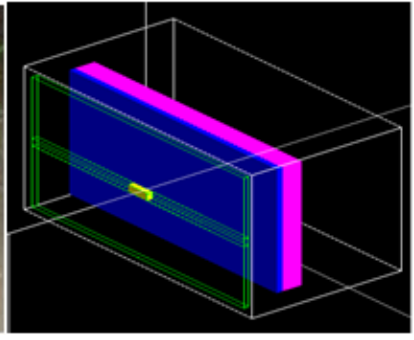

(b)

Figure 3. (a) One silicon detector unit. (b) A parallel configured dual planar silion-NaI Compton imaging system (the yellow cube is a stack of 10 individual $1 \mathrm{~mm}$ think silicon scatter detector, and the blue cube is $\mathrm{NaI}$ crystal) .

together to increase the scatter detector sensitivity. Therefore, the total sensitive volume is $44.8 \times 22.4 \times 10 \mathrm{~mm}^{3}$, and energy resolution is $1 \mathrm{keV}$. The absorption detector is a NaI Argus Anger camera imaging head without collimator. As demonstrated in figure 3(b), the two planar detectors are parallel and centers of both detectors are aligned on axis.

2.3.2. Statistical model of Compton imaging system. Unlike derivation of the Anger camera system model by simulating or measuring a series of point-spread functions, for the relatively complex Compton imaging system, the system model must be derived by mathematical approximation for each physical process involved including the estimation of position resolution, energy resolution and the effect of Doppler broadening by calculation of the Compton differential cross section. Therefore, accurate modeling of the Compton imaging system is necessary to correctly calculate the detection sensitivity and transition probabilities, which determine the estimated quality of image reconstruction and the accuracy of performance evaluation by the M-UCRB. However, calculating a rigorous statistical model suffers from the computational complexity of the multidimensional integration involved. The tradeoff between a practical calculation and accurate estimation needs to be taken into account. In this section, we investigate an approximate statistical model for the configuration of the proposed Compton camera that accurately describes the sequence of physical processes.

The sequence of physical processes involved in one detected event in a Compton imaging system is

(1) A $\gamma$-ray photon is emitted from source position $\underline{x}_{0}$ in the object with initial energy $e_{0}$ and direction $\Omega_{1}$.

(2) The emitted photon escapes from the object.

(3) The escaped photon is directed toward the first (scatter) detector.

(4) At location $\underline{z}_{1}$ of the first detector, the photon Compton scatters from an electron and energy $e_{1}$ is deposited in the first detector and the scattered photon escapes from the first detector in direction of solid angle $\Omega_{2}$. If the energy $e_{1}$ is larger than the detection threshold, the location $\underline{z}_{1}$ and energy $e_{1}$ are recorded.

(5) If the second detector lies within $\Omega_{2}$, the scattered photon passes through an attenuating medium or air between the first detector and second detector.

(6) The scattered photon with energy $\left(e_{0}-e_{1}\right)$ strikes the second detector and may be absorbed in the second detector. The energy $e_{2}$ and position $\underline{z}_{2}$ are measured by the 


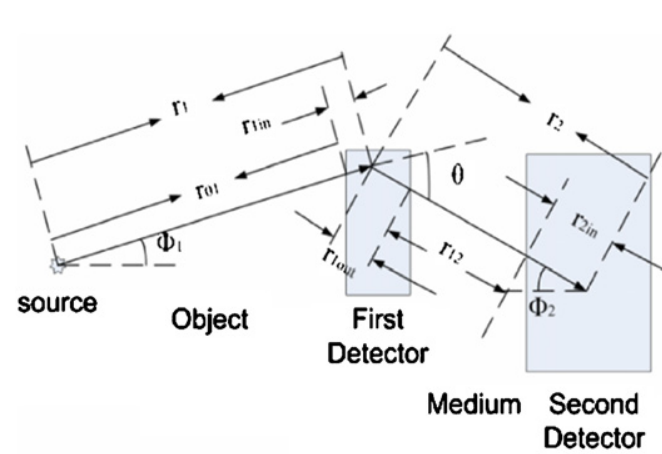

(a)

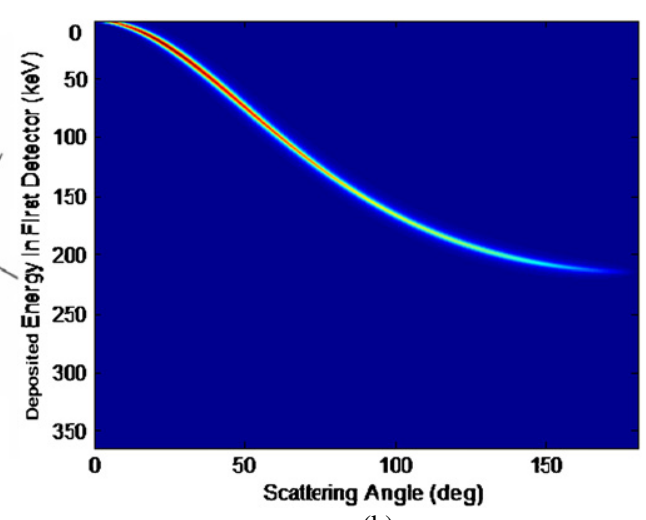

(b)

Figure 4. (a) Geometry and parameter definition of Compton imaging system with two parallel block detectors. $\Phi_{1}$ is the zenith angle of source photon; $\Phi_{2}$ is the zenith angle of scattered photon; $\theta$ is the Compton scatter angle; $r_{1}=r_{01}+r_{\text {in }} ; r_{2}=r_{1 \text { out }}+r_{12}+r_{2}$ in. (b) The Joint probability density functions that includes Doppler broadening and first detector energy resolution of a crystalline silicon detector for $364.4 \mathrm{keV}$ incoming photons (the position resolution of the silicon detector is $1.4 \mathrm{~mm}$; energy resolution of silicon detector is $1 \mathrm{keV}$; The position resolution of the second detector is $4 \mathrm{~mm}$, and the distance between the two detectors is $100 \mathrm{~mm}$ ).

second detector. The energy $e_{2}$ may or may not equal to $\left(e_{0}-e_{1}\right)$. This depends on whether the scattered photon is fully absorbed by the second detector or perhaps scatters and escapes.

Therefore, the transition probabilities can be evaluated as

$$
\begin{aligned}
p(Y / i)=\frac{1}{8 \pi^{2}} & \frac{u_{c s-1}\left(e_{0}\right)}{u_{t-1}\left(e_{0}\right)} \mathrm{e}^{-u_{t-o}\left(e_{0}\right) r_{01}}\left(1-\mathrm{e}^{-u_{t-1}\left(e_{0}\right) r_{\text {lin }}}\right) \mathrm{e}^{-u_{t-1}\left(e_{2}\right) r_{1 \text { out }}} \\
& \times \mathrm{e}^{-u_{t-m}\left(e_{2}\right) r_{12}}\left(1-\mathrm{e}^{-u_{t-2}\left(e_{2}\right) r_{2 \text { in }}}\right) p\left(\theta, e_{1}^{\prime}\right) \frac{\cos \Phi_{1}}{r_{1}^{2}} \frac{\cos \Phi_{2}}{r_{2}^{2}}
\end{aligned}
$$

Where $\mu_{\mathrm{cs}}$ is the silicon linear attenuation coefficient for the Compton process, and $\underline{\mu}_{\mathrm{t}}$ is the total linear attenuation coefficient for different materials. The definition of other parameters is described in figure $4(\mathrm{a})$.

In equation (20), $p\left(\theta, e_{1}^{\prime}\right)$ is the Compton scattering based joint probability density function (JPDF) combined with energy and position measurement noise. The density is evaluated by interpolation from the precalculated 2D table indexed by Compton scatter angle $\theta$ and measured deposited energy $e_{1}^{\prime}$ in the first detector. As shown in figure 4(b), The matrix for crystalline silicon detecting $364.4 \mathrm{keV}$ photons is indexed by the scattering angle (horizontal axis) and deposited energy (vertical axis), which is the combined effect of Doppler broadening, detector energy resolution and spatial resolution.

\section{Results and analysis}

In this section, the performance of the Compton imaging system and the Anger Camera with HEGP collimator for imaging $364.4 \mathrm{keV}$ photons are analyzed and compared using the M-UCRB, reconstructed images using MLEM algorithms, and detection sensitivity. 


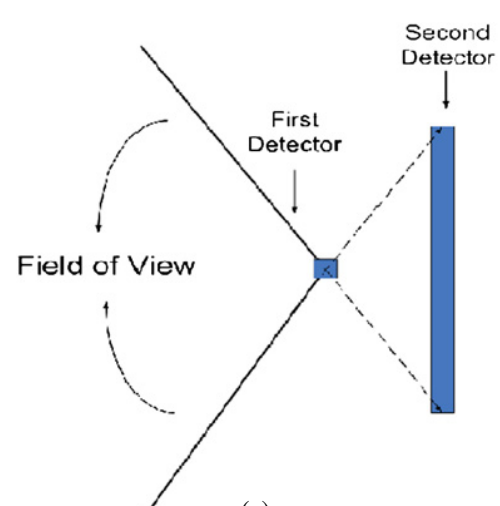

(a)

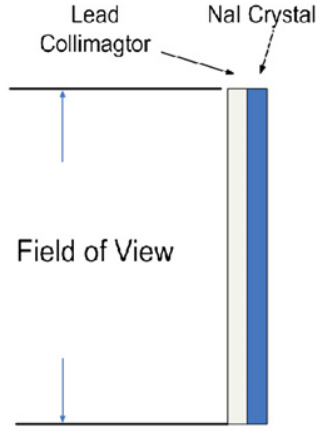

(b)

Figure 5. (a) The Field of View of one pixel of the Compton imaging system with scattering angle from 0 degree to 180 degree scattering angle (top view). (b) The Field of View of the collimated Anger Camera (top view).

\subsection{Comparison of field of view}

Top views of the Compton imaging system and conventional collimated Anger camera are displayed in figures 5(a) and (b), respectively. The size of collimated Anger camera determines the size of the field of view (FOV), whereas the FOV of the Compton imaging system primarily depends on the geometry of the overall system and can be considerably larger than the first detector as illustrated by Rogers and Clinthorne (2004). Essentially, each first detector element acquires a cone-beam projection and can be considered analogous to a 'virtual pinhole' after either explicit Compton decoding or implicit decoding as part of the image reconstruction process.

\subsection{Performance comparison using $M-U C R B$}

3.2.1. The two-dimensional thin disk object. The object used for the M-UCRB calculations was a $26 \mathrm{~cm}$ diameter disk having uniform activity. The reconstructed image was quantized into $65 \times 65$ pixel array with pixel size $0.4 \mathrm{~cm}$ by $0.4 \mathrm{~cm}$. The disk faced the imaging system and the center of the disk was aligned with the center of the detector. It was located $10 \mathrm{~cm}$ from the collimator of the Anger camera and $10 \mathrm{~cm}$ from the first detector of the Compton imager, in which the distance between the first and second detectors was also $10 \mathrm{~cm}$. The center pixels of the disk were selected for evaluation.

Three M-UCRB curves and the ratio of the Compton to the Anger camera M-UCRB curves for the same number of events are displayed in figure 6(a) and (b), respectively. The Anger camera with HEGP collimator is illustrated for two mathematical models. The Anger camera modeled with a pure Gaussian response according to the specifications of the Argus Anger Camera, which is 12.6 mm FWHM (Han 2008), has better performance when the FWHM of the desired PSF is larger than $1.1 \mathrm{~cm}$. The ratio of M-UCRB on variance is around 9. This indicates that the sensitivity of the Compton Imaging system must be about 9 times greater than the Anger Camera to obtain the same performance. However, when the Gaussian plus exponential tails are modeled according to 16171819 for a target PSF less than $1.3 \mathrm{~cm}$, the Compton imaging system significantly outperforms the collimated Anger camera for the same number of detected events. Even at the lowest reconstructed resolution, the M-UCRB 


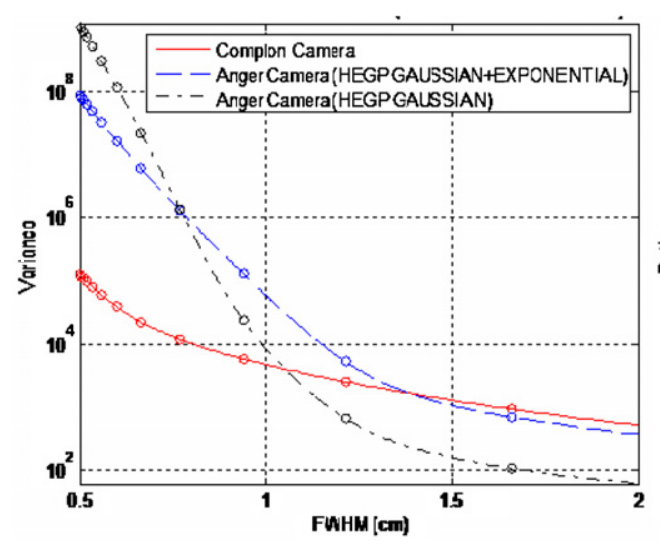

(a)

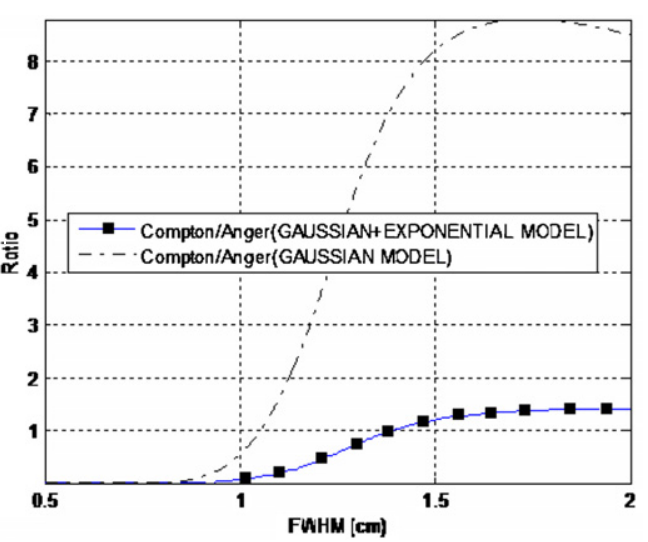

(b)

Figure 6. (a) M-UCRB curves of same number of events for a Compton imaging system and an Anger camera with HEGP collimator for imaging $364.4 \mathrm{keV}$ photons. The variance in center pixel intensity of the $26 \mathrm{~cm}$ diameter uniform source disk is evaluated. The Anger Camera with HEGP response is modeled as a pure Gaussian function $(\mathrm{FWHM}=12.6 \mathrm{~mm})$ and as a Gaussian plus exponential tails $\left(A_{\text {Gauss }}=0.0164, \sigma_{\text {Gauss }}=5.22, A_{\exp }=0.0021\right.$, and $\left.\lambda_{\exp }=0.0235\right)$ that more accurately represents the collimator response for $360 \mathrm{keV}$ photons. (b) The variance bound ratio of M-UCRB for a Compton imaging system over Anger Camera with 2 HEGP collimator models.

curves for the two systems with identical sensitivity are close with a ratio of approximately 1.1 for the same number of events.

3.2.2. The three-dimensional cylindrical object. The reconstruction domain for a 3D object study was $20 \mathrm{~cm} \times 20 \mathrm{~cm} \times 20 \mathrm{~cm}$ quantized into $65 \times 65 \times 65$ pixels array. Each pixel was a $0.31 \mathrm{~cm}$ cube. The simulated object was a uniform cylinder with diameter of $20 \mathrm{~cm}$. The central axis of the cylinder is parallel to the surface of the detector, and the distance between the axis and detector surface is $14 \mathrm{~cm}$. The object was rotated in steps of $1^{\circ}$ over a total of $360^{\circ}$ for calculating the 3D Fisher information matrix.

The central pixel in the object was selected to calculate M-UCRB and, as for the 2D case, the number of events for calculating the FIM was the same for both systems. As shown in figure 7, the Compton imaging system achieves a substantially lower bound on variance than the collimated Anger camera with HEGP per detected photon irrespective of desired resolution. Compared with the collimated Anger camera from figure 7, the minimum ratio between the two bound curves is about 1.9 at around $1.4 \mathrm{~cm}$ (FWHM). For the 3D case, both line integrals and cone integrals are used to reconstruct the image and calculate the FIM for the Anger camera and Compton imaging systems, respectively. Compared with the M-UCRB in the $2 \mathrm{D}$ case, the effects of these integrals introduce relatively more degradation of the Anger camera performance than for the Compton imaging system.

\subsection{Comparison of detection sensitivity}

The detection efficiency or sensitivity is defined as the ratio of the number of accepted detected events to the total number of emitted gamma-rays from the source. The sensitivity is determined by the energy of the photons, system geometry and detector and collimator material. As shown in figure 8(a), the detection sensitivities at a different source to detector distance of the Anger camera with HEGP collimator are evaluated by Monte Carlo Simulation. 


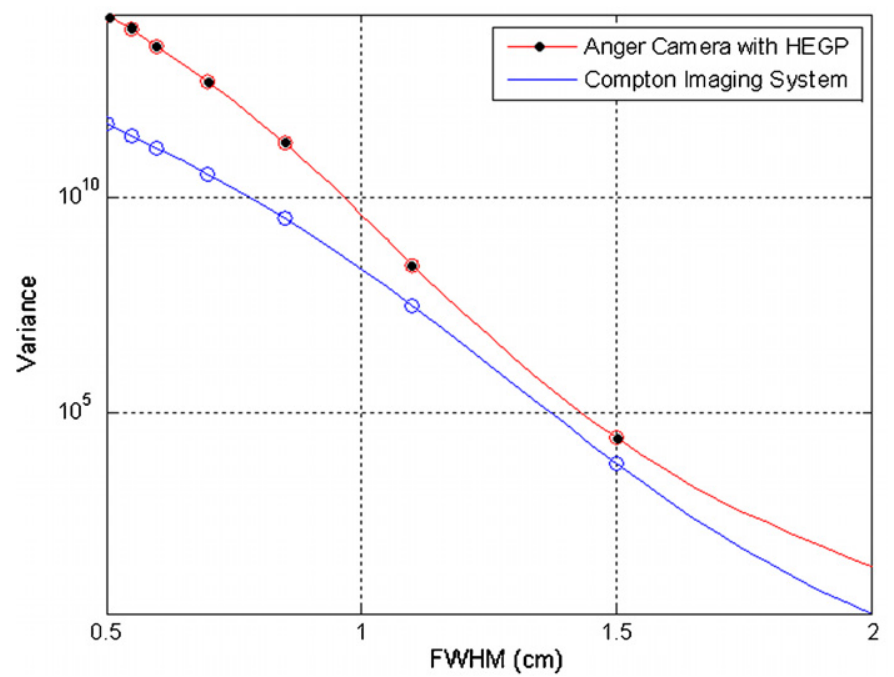

Figure 7. M-UCRB curves for the central element for a Compton imaging system and an Anger camera with HEGP for detecting $364.4 \mathrm{keV}$ photons for the same number of counts. The source object is a 3D cylinder with diameter of $20 \mathrm{~cm}$ and height of $20 \mathrm{~cm}$.

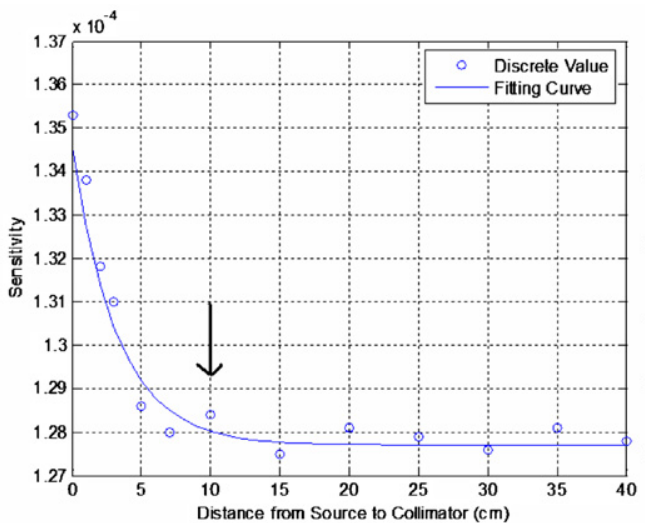

(a)

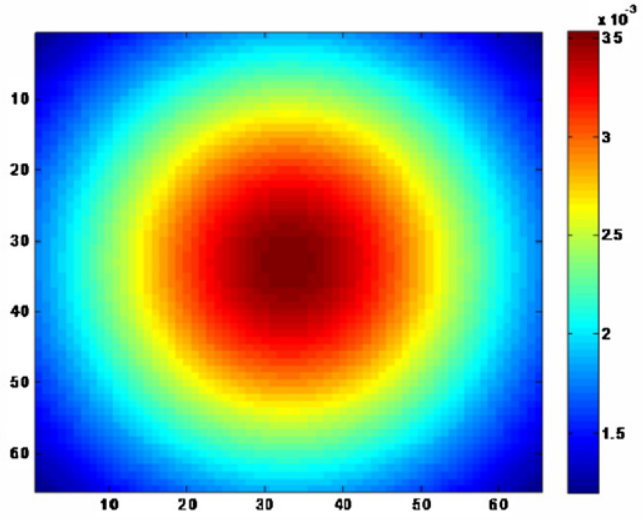

(b)

Figure 8. (a) The detection sensitivity value and curve for detecting a I ${ }^{131}$ point source by ARGUS Anger Camera with HEGP collimator. (b) Calculated detection sensitivity map for the proposed Compton imaging system. The source plane is located at $10 \mathrm{~cm}$ to the first detector.

For a given distance from the source to the detector, the sensitivity of Compton imaging system is not uniform. Using the system model of Compton imaging system presented, the histogram of calculated sensitivities is displayed in figure 8 (b) for a $65 \times 0.31 \mathrm{~cm}$ by $65 \times 0.31 \mathrm{~cm}$ object located at $10 \mathrm{~cm}$ from the surface of the silicon detector. The maximum sensitivity for the center point is around $3.45 \times 10^{-3}$, and the average sensitivity is around $2.34 \times 10^{-3}$. Therefore, at a $10 \mathrm{~cm}$ source to detector distance, the average detection sensitivity of Compton imaging system is about 20 times that of the Anger camera with the HEGP collimator. 


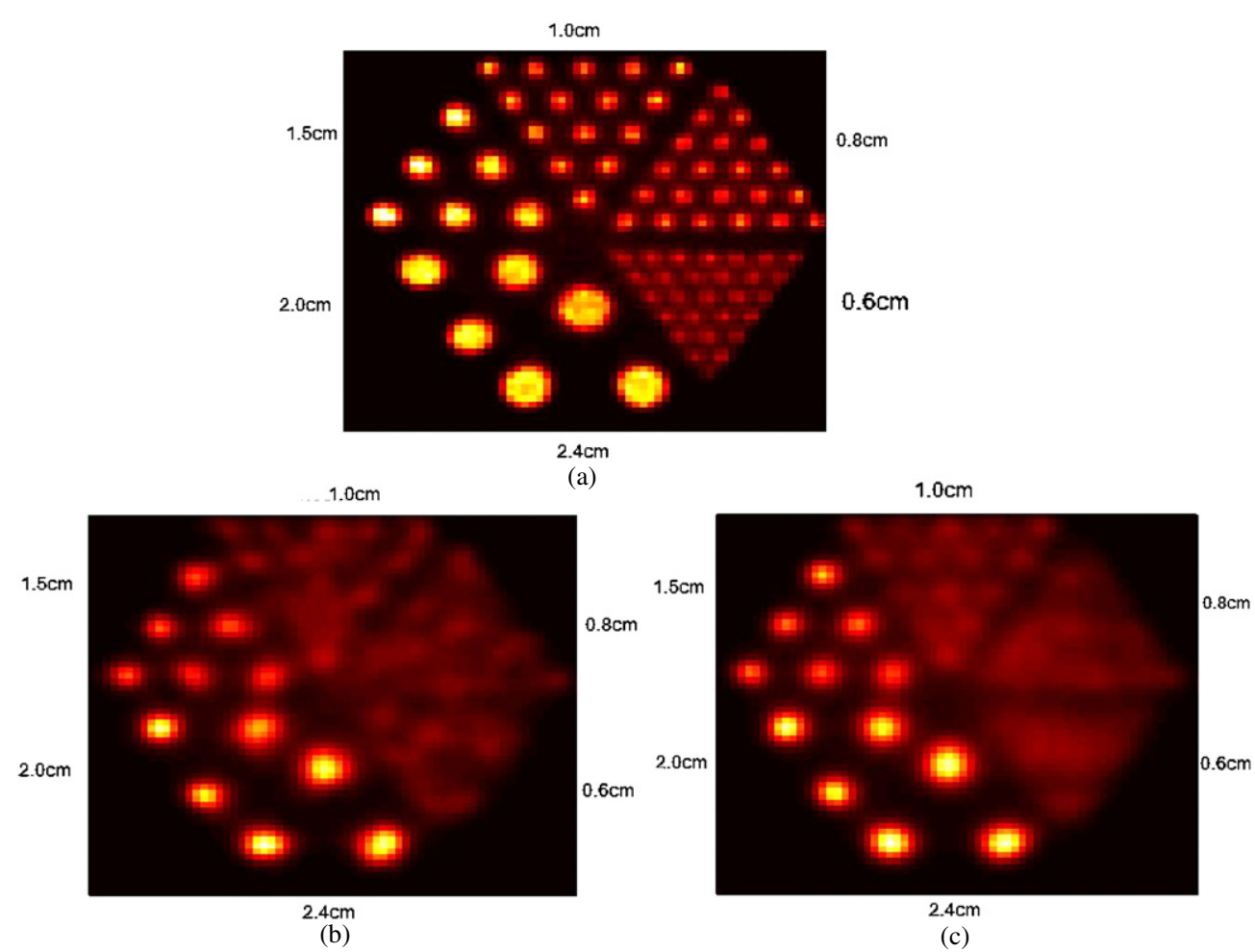

Figure 9. Reconstructed images using 100 iterations of MLEM for the hot spot disk. Hot spots are $0.6 \mathrm{~cm}, 0.8 \mathrm{~cm}, 1.0 \mathrm{~cm}, 1.5 \mathrm{~cm}, 2.0 \mathrm{~cm}$ and $2.4 \mathrm{~cm}$ diameter. (a) Compton imaging system with 3 million events. (b) Anger Camera with 0.2 million events (equal imaging time). (c) Anger Camera with 3 million events.

\subsection{Performance comparison using restored $2 D$ images}

Although the M-UCRB analysis provides valuable information in regards to the relative performance of imaging systems over a range of resolutions, the analysis in this study has its limits. In particular the resolution-noise tradeoff was only evaluated at a single point in a large uniformly emitting disk or cylinder. While performance at this point is likely representative of other locations in the image, there is the chance that it is not. Furthermore, calculations of the M-UCRB used several approximations as noted in sections 2.1.2 and 2.1.3. Because of these issues, we corroborated bound predictions with maximum-likelihood reconstructions from a $2 \mathrm{D}$ resolution phantom for each system.

A simulated two-dimensional thin sheet phantom with ${ }^{131} \mathrm{I}$ was placed $10 \mathrm{~cm}$ from the front surface of the two detectors. The diameters of hot spots on the phantom are $0.6 \mathrm{~cm}$, $0.8 \mathrm{~cm}, 1 \mathrm{~cm}, 1.5 \mathrm{~cm}, 2.0 \mathrm{~cm}$ and $2.4 \mathrm{~cm}$. The intensities of hot spots are uniform and the background activity is zero. Images with 65 by $650.308 \mathrm{~cm}$ pixels were reconstructed by 100 iterations of MLEM for both the Compton imaging system and the Anger camera with HEGP collimator. As shown in figure 9(a), three million events were acquired for the Compton imaging system and its image was reconstructed by list-mode MLEM. Since the detection sensitivity of the Compton imaging system is about 20 times of the collimated Anger camera, 200 thousand events were acquired by the Anger Camera with HEGP collimator, and its 
reconstructed image is displayed in figure 9(b). For comparison, the image for Anger Camera with HEGP with three million events is also shown in figure 9(c). Clearly, the Compton imaging system performance is substantially better than the conventional Anger camera, even for the same number of events. In particular, hot spots less than $1.0 \mathrm{~cm}$ diameter cannot be reconstructed for the Anger camera with HEGP, since information provided by this imaging system is not sufficient to recover such small hot spots. However, even the smallest hot spots for the Compton system are clearly defined. For equal imaging time the collimated camera image is much noisier and more poorly defined.

\section{Discussion and conclusion}

The results of both the M-UCRB and 2D image simulations show that the Compton imaging system studied outperforms the collimated Anger camera for imaging higher energy, $364.4 \mathrm{keV}$, photons. Even though the enhancement can be observed directly in figure 9 by comparison of restored images, the qualitative analysis is not sufficient to demonstrate improvement at every desired reconstructed resolution since it is possible to trade resolution against noise. The M-UCRB analysis helps to quantify this tradeoff for each imaging system and moreover, it provides the limiting variance based only on the intrinsic properties of the imaging system, which eliminates the confounding influence of the image reconstruction algorithm. With this technique, the lowest achievable variance at each specific target PSF for different imaging systems was compared. Limitations of the M-UCRB analysis still exist due to use of approximations to reduce computation and the fact that the ability to quantify small changes in source intensity at the central point in a large, uniformly emitting disk was assumed to be representative of other points in the image. The qualitative information provided by reconstructions in figure 9 provides confidence that the point evaluated for the M-UCRB analysis is indeed representative.

In the 2D analysis, for the same number of imaged events, noise performance of the Compton system is better than using both Anger camera collimator models when the desired reconstructed PSF width is below $\sim 11 \mathrm{~mm}$ FWHM. Although the relative advantage decreases below unity above $11 \mathrm{~mm}$ for the pure Gaussian collimator model and above $14 \mathrm{~mm}$ for the more realistic Gaussian-exponential model, figure 8 shows that the Compton system model can have a 20 -fold advantage in sensitivity. This results in an overall performance advantage over both collimator models throughout the full 5-20 mm range of reconstructed resolution. For 3D, the Compton advantage proves even more compelling. On the basis of the same number of detected events, the limiting noise in the Compton system is uniformly lower than the conventionally collimated system regardless of resolution. The Compton efficiency advantage results in an additional 20 times decrease in noise.

Although the predicted performance of the Compton camera is superior to the collimated Anger camera at the energies of ${ }^{131} \mathrm{I}$, the performance advantage decreases rapidly at lower energies. This is due both to the fact that collimators at lower energies are more efficient for a given resolution due to the rapid increase in the photoelectric absorption cross section and the fact that Compton performance degrades rapidly for decreasing energies due to increased scattering angle uncertainty for a given measured energy. As noted by previous investigators, Compton cameras require significantly increased efficiency over conventional collimation at $140 \mathrm{keV}$ to achieve break-even performance (Hua 1999). The large volume of low-noise silicon detectors, needed to achieve such efficiency is likely impractical at present. In contrast, the volume of silicon detector required to achieve the 20 -fold sensitivity advantage noted above — while not inexpensive—is readily achievable with current technology. Furthermore, 
our predictions show that each detected photon in a Compton camera carries more information at high resolution than the conventionally collimated systems.

Despite the promise of significant performance improvements over collimated Anger cameras at higher energies, there remain many challenges in constructing a Compton camera practical for medical imaging. In particular, the camera requires low-noise silicon detectors for scattering incident photons. This requirement has largely been met through the use of silicon pad detectors and readout technology that routinely achieve $1.4 \mathrm{keV}$ FWHM energy resolution and are capable of reliable triggering at less than $10 \mathrm{keV}$. Another challenge is the need for processing high event rates in the scattering and absorption detectors as well as the corresponding need for good timing resolution (10-20 ns FWHM) to keep random coincidence rates low. This has not been achieved with our experimental setup that uses a modified Anger scintillation camera as the absorption detector but is certainly possible using alternative technologies such as LSO-based PET detectors. Finally, the sheer computation required for image reconstruction-especially in $3 \mathrm{D}$-is an issue but the almost relentless march of Moore's Law in conjunction with coarse-grained parallel computing methods may well eliminate it as such in the near future.

\section{Acknowledgment}

This research was supported by Grant R01-EB430 from National Institutes of Health (NIH).

\section{References}

ADAC Laboratories ARGUS System Specifications 1-6

Anger H O 1958 Scintillation camera Rev. Sci. Instrum. 29 27-33

Anger H O 1964 Scintillation camera with multichannel collimators J. Nucl. Med. 65 515-31

Berger J O 1985 Statistical Decision Theory and Bayesian Analysis (New York: Springer)

Compton A H 1923 A quantum theory of the scattering of x-rays by light elements Phys. Rev. 21 483-502

Davis P J 1994 Circulant Matrices (New York: Chelsea)

Evans R D 1982 Atomic Nucleus (New York: Krieger)

Fessler J A and Rogers W L 1996 Spatial resolution properties of penalized-likelihood image reconstruction: Spaceinvariant tomographs IEEE Trans. Med. Imaging. 5 1346-58

Han L 2008 Statistical performance evaluation, system modeling, distributed computation, and signal pattern matching for a Compton medical imaging system PhD Dissertation Deparment of Biomedical Engineering, The University of Michigan, Ann Arbor, MI, USA

Hero A O, Fessler J A and Usman M 1996 Exploring estimator bias-variance tradeoff using the uniform CR bound IEEE Trans. Signal Processing 44 2026-41

Hua C H, Clinthorne N H, Wilderman S J, Leblanc J W and Rogers W L 1999 Quantitative evaluation of information loss for Compton cameras IEEE Trans. Nucl. Sci. 465 587-93

Jan S, Santin G and Strul D 2004 GATE: a simulation toolkit for PET and SPECT Phys. Med. Bio. 49 $4543-61$

Kay S M 1993 Fundamentals of Statistical Signal Processing: Estimation Theory, Volume I (Upper Saddle River, NJ: Prentice Hall)

Knoll G F 2000 Radiation Detection and Measurement (New York: Wiley)

Kragh T J 2002 Tradeoffs and limitations in statistically based image reconstrustruction problems PhD Dissertation Department of Electrical Engineering and Computer Science, The University of Michigan, Ann Arbor, MI, USA

Leblanc J W 1999 A Compton camera for low energy gamma ray imaging in nuclear medicine applications $P h D$ Dissertation Department of Nuclear Engineering and Radiological Sciences, The University of Michigan, Ann Arbor, MI, USA

Leblanc J W, Clinthorne N H, Hua C-H, Nygard E, Rogers W L, Wehe D K, Weilhammer P and Wilderman S J 1998 C-SPRINT: A prototype Compton camera system for low energy gamma ray imaging IEEE Trans. Nucl. Sci. 45 943-9

Meng L J and Clinthorne N H 2004 A modified uniform Cramer-Rao bound for multiple pinhole aperture design IEEE Trans. Medical Imaging 23 896-902 
Meng L J and Wehe D K 2003 Feasibility study of using hybrid collimation for nuclear environmental imaging IEEE Trans. Nuclear Science 55 1103-10

Ordonez C E, Bolozdynya A and Chang W 1997 Doppler broadening and energy uncertainties in Compton cameras IEEE Nucl. Sci. Symp. Conf. Rec. 2 1361-65

Parra L and Barrett H H 1998 List-mode likelihood: EM algorithm and image quality estimation demonstrated on 2D PET IEEE Trans. Medical Imaging 17 228-35

Philips G W 1995 Gamma-ray imaging with Compton cameras Nucl. Imaging Methods Phys. Res. B 99 674-77

Press W H, Teukolsky S A, Vetterling W T and Flannery B P 2002 Numerical Recipes in C: the art of scientific computing (Cambridge: Cambridge University Press)

Rogers W L, Clinthorne N H and Bolozdynia A 2004 Ch 19, Compton cameras for Nuclear Medical Imaging Emission Tomography: The Fundamentals of PET and SPECT ed M N Wernick and J N Aarsvold (New York: Academic) pp 383-420

Singh M 1983 An electronically collimated gamma camera for single-photon emission computed tomography. Part I: Theoretical considerations and design criteria. Med. Phys. 10 421-27

Solomon S J and Ott R J 1988 Gamma ray imaging with silicon detectors-a Compton camera for radionuclide imaging in medicine Nucl. Instrum. Methods Phys. Res. A 273 787-92

Todd R W, Nightingale J M and Everett D B 1974 A proposed Gamma camera Nature. 251 132-4

Usman M 1994 Biased and unbiased Cramer-Rao bounds: computation and application PhD Dissertation (Ann Arbor: The University of Michigan)

Van Trees H L 2001 Detection, Estimation, and Modulation Theory, Part I (New York: Wiley) 